\title{
A method used to overcome polarization effects in semi-polar structures of nitride light-emitting diodes emitting green radiation
}

\author{
Seweryn Morawiec • Robert P. Sarzała • \\ Włodzimierz Nakwaski
}

Received: 3 December 2012 / Accepted: 26 January 2013 / Published online: 22 February 2013

(C) The Author(s) 2013. This article is published with open access at Springerlink.com

\begin{abstract}
Polarization effects are studied within nitride light-emitting diodes (LEDs) manufactured on standard polar and semipolar substrates. A new theoretical approach, somewhat different than standard ones, is proposed to this end. It is well known that when regular polar GaN substrates are used, strong piezoelectric and spontaneous polarizations create built-in electric fields leading to the quantumconfined Stark effects (QCSEs). These effects may be completely avoided in nonpolar crystallographic orientations, but then there are problems with manufacturing InGaN layers of relatively high Indium contents necessary for the green emission. Hence, a procedure leading to partly overcoming these polarization problems in semi-polar LEDs emitting green radiation is proposed. The (1122) crystallographic substrate orientation (inclination angle of $58^{\circ}$ to $c$ plane) seems to be the most promising because it is characterized by low Miller-Bravais indices leading to highquality and high Indium content smooth growth planes. Besides, it makes possible an increased Indium incorporation efficiency and it is efficient in suppressing QCSE. The $\mathrm{In}_{0.3} \mathrm{Ga}_{0.7} \mathrm{~N} / \mathrm{GaN} \mathrm{QW}$ LED grown on the semipolar (1122) substrate has been found as currently the optimal LED structure emitting green radiation.
\end{abstract}

\section{Introduction}

Nitride based III-V semiconductors, i.e., GaN, InN, and $\mathrm{AlN}$ and their ternary and quaternary alloys, have recently

S. Morawiec · R.P. Sarzała · W. Nakwaski $(\bowtie)$

Photonics Group, Institute of Physics, Lodz University

of Technology, ul. Wolczanska 219, 90-924 Lodz, Poland

e-mail:wlodzimierz.nakwaski@p.lodz.pl attracted a great interest of research centers due to their possible applications in manufacturing visible and even ultraviolet light emitting diodes (LEDs). These materials, however, differ significantly from most of other III-V semiconductors, which leads for example to problems with obtaining efficient nitride sources of green radiation ("green gap" effect) [1-3]. Their special properties are connected with their wurtzite crystal structure, distinctly different from the zinc blende structure of most of other III-V semiconductors. In Fig. 1, characteristic planes and axes of this crystallographic structure are shown. Layers of most of nitride devices are usually grown along the $c$ polar axis. Planes parallel to this axis, i.e., perpendicular to the $c$ plane, are electrically neutral (nonpolar), because they contain the same number of both anions and cations, whereas planes between the polar $c$ and any nonpolar orientations are called semipolar ones $[5,6]$. Crystallographic plane is usually defined by its inclination angle $\vartheta$ between the crystallographic direction $c$ and the axis perpendicular to this plane (Fig. 2).

In $\mathrm{GaN}$, the $c / a$ ratio is equal to 1.626 [8], whereas its value for the ideal wurtzite structure equals about 1.633 [9], where $c$ and $a$ are the lattice constants in the $c$ and $a$ crystallographic directions, respectively. Therefore, the GaN crystal is compressed in the $c$ direction. It results in some shifting of positive and negative charges, which is always directed along the $c$ axis [10], and is called the spontaneous polarization [9] —an intrinsic crystal property. Possible further crystal stresses lead to an additional change of the $c / a$ ratio, which results in the piezoelectric polarization. In quantum wells (QWs), it leads to strong build-in electric fields, and consequently to the quantum-confined Stark effect (QCSE): electrons and holes are shifted in opposite directions (compare peak positions of electron and heavy-hole wave functions determined for the first QW energy levels shown in Fig. 3). Then overlapping of their wave functions 


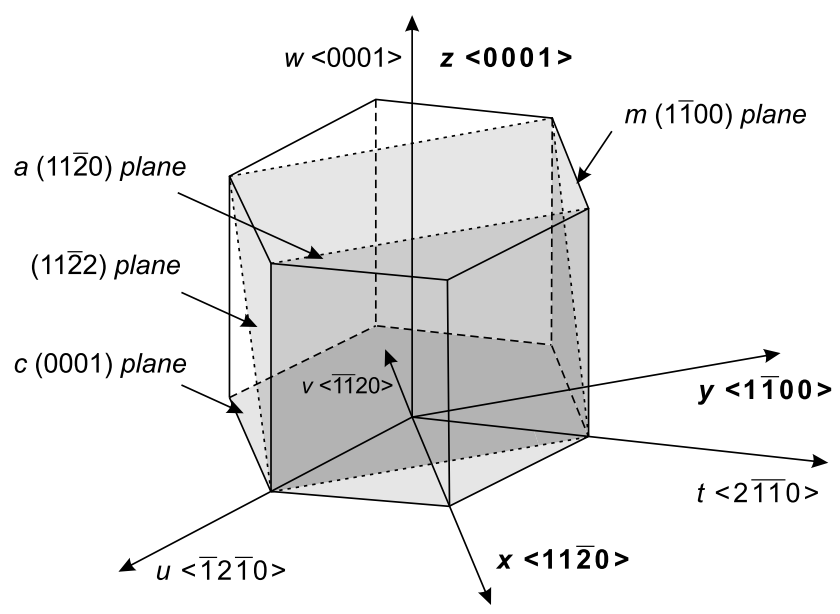

Fig. 1 Possible growth planes and crystallographic directions in the hexagonal unit cell (on basis of [4])

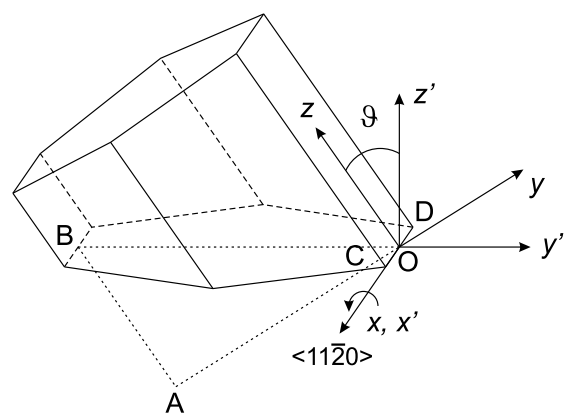

Fig. 2 Wurtzite structure in the semi-polar orientation with the local $0 x y z$ and the global $0^{\prime} x^{\prime} y^{\prime} z^{\prime}$ co-ordinate systems. $\vartheta$-inclination angle. The inclination axis is along the $<1120>$ direction. (On the basis of [7])

is strongly decreased, which is discussed in Sect. 5. Moreover, QW barriers are effectively reduced enabling more intense escape currents from QWs, especially at higher temperatures.

In most of commercially available substrates, the (0001) $c$ plane is used to manufacture nitride devices, which leads to mostly unprofitable polarization effects. Recently, however, relatively thick $c$-oriented $\mathrm{GaN}$ substrates have been reported (e.g., [11]). Then proper slicing may be used to produce nonpolar or semipolar native GaN substrates [2, 5].

Desired emission of green radiation requires in nitride devices relatively high Indium contents. In nonpolar LEDs, however, an increase in an Indium content results in degraded crystal quality because of required low growth temperatures [2]. Efficiency of some similar semipolar LEDs also decreases for longer wavelengths, but to much less extent. Therefore, the semipolar substrate orientation is considered (e.g., [6]) in our simulation. Quality of layers grown on semipolar substrates has been found to be similar to that of layers grown on nonpolar substrates [7]. However, highquality and high Indium content smooth growth planes may

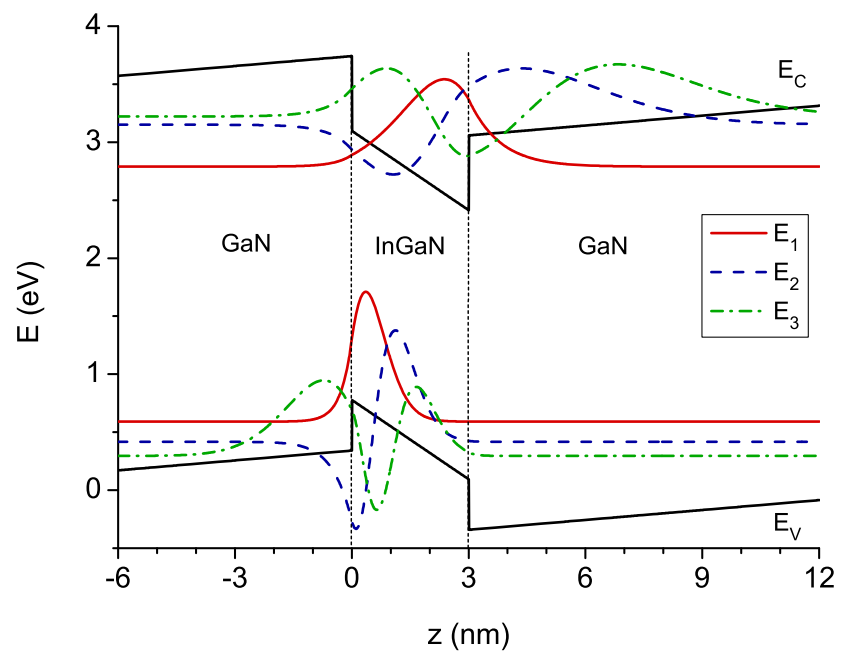

Fig. 3 Band model of the 3-nm $\operatorname{In}_{0.3} \mathrm{Ga}_{0.7} \mathrm{~N} / \mathrm{GaN}$ QW presenting wave functions of trapped carriers

be then obtained with low values of the Miller-Bravais indices [7]. Besides, also partial reduction of polarization effects may be then obtained.

Typical InGaN/GaN LEDs grown on the $c$-oriented substrate exhibit unprofitable strong polarization effects because both spontaneous and piezoelectric polarizations are increased with an increase in the Indium content in InGaN. They create serious problems especially in highly strained InGaN/GaN QWs with high Indium content designed to emit green radiation $[1,2]$. However, it is shown in Sect. 4 that polarization effects are excluded not only for the total polarization reduced to zero, but also for equal polarizations in each layer of LED structure. Anyway, their unprofitable impact on LEDs operation may be at least partly reduced with the aid of a careful technology.

\section{Polarization effects in the semi-polar crystal structure}

A typical nitride LED structure is composed of the following layer sequence: $n$-type substrate, QW barrier, QW, QW barrier, electron-blocking layer, and $p$-type layer. Let us consider its manufacturing on a semipolar substrate. The local coordinate $0 x y z$ system is correlated with the substrate crystalline structure (its $z$ axis is directed along the $c$ crystalline axis-see Fig. 2), whereas the analogous global $0 x^{\prime} y^{\prime} z^{\prime}$ system has the $z^{\prime}$ axis at the inclination $\vartheta$ angle with respect to the $z$ axis and $x^{\prime}$ axis along the $x$ axis. The depths of the InGaN/GaN QW within the conduction and valence bands are determined using approach of Sharma and Towe [3] and assuming its ratio (band offset ratio) equal to 1.48.

Let us consider the semipolar wurtzite structure shown in Fig. 2. Following the approach reported by Romanov et al. [7], misfit parameters along the $x^{\prime}$ and $y^{\prime}$ axes may be 
expressed as

$\varepsilon_{x}=\frac{a_{\mathrm{S}}-a_{\mathrm{L}}}{a_{\mathrm{L}}}$

$\varepsilon_{y}=\frac{a_{\mathrm{S}} c_{\mathrm{S}}-\sqrt{\left(a_{\mathrm{L}} c_{\mathrm{S}}\right)^{2} \cos ^{2} \vartheta+\left(a_{\mathrm{S}} c_{\mathrm{L}}\right)^{2} \sin ^{2} \vartheta}}{\sqrt{\left(a_{\mathrm{L}} c_{\mathrm{S}}\right)^{2} \cos ^{2} \vartheta+\left(a_{\mathrm{S}} c_{\mathrm{L}}\right)^{2} \sin ^{2} \vartheta}}$

where $a_{\mathrm{S}}$ and $c_{\mathrm{S}}$ are hexagonal parameters of the substrate material whereas $a_{\mathrm{L}}$ and $c_{\mathrm{L}}$ are analogous parameters of the layer material. For the layer thickness below, its critical value for formation of misfit dislocations, the misfit between the substrate and the layer is accommodated by the elastic strain within the layer. Assuming no in-plane shear mismatch at the interface, some components of the strain tensor may be found directly:

$\varepsilon_{x^{\prime} x^{\prime}}=\varepsilon_{x} \quad \varepsilon_{y^{\prime} y^{\prime}}=\varepsilon_{y} \quad \varepsilon_{x^{\prime} y^{\prime}}=0$

Besides, from the condition for the mechanical equilibrium between the substrate and the layer, some components of the stress tensor in the global $0 x^{\prime} y^{\prime} z^{\prime}$ co-ordinate system may be also deduced:

$\sigma_{z^{\prime} z^{\prime}}=\sigma_{x^{\prime} z^{\prime}}=\sigma_{y^{\prime} z^{\prime}}=0$

Determination of the remaining components of the strain and the stress tensors requires an application of the Hook's law:

$\sigma_{i j}=\sum_{k, l=1}^{3} C_{i j k l} \varepsilon_{k l}$

where $C_{i j k l}$ are elements of the fourth-rank elastic stiffness tensor, whereas $\sigma_{i j}$ and $\varepsilon_{k l}$ are elements of the second-rank stress and strain, respectively, tensors. Using the Voigt notation [12] for the wurtzite III-nitrides, there exist only five independent stiffness coefficients and twelve non-zero $C_{i j}$ elements [7, 10]:

$C_{i j}=\left[\begin{array}{cccccc}C_{11} & C_{12} & C_{13} & 0 & 0 & 0 \\ C_{12} & C_{11} & C_{13} & 0 & 0 & 0 \\ C_{13} & C_{13} & C_{33} & 0 & 0 & 0 \\ 0 & 0 & 0 & C_{44} & 0 & 0 \\ 0 & 0 & 0 & 0 & C_{44} & 0 \\ 0 & 0 & 0 & 0 & 0 & \frac{C_{11}-C_{12}}{2}\end{array}\right]$

where values of the $C_{i j}$ elements have been given in [8]. For a free crystal, all rotation strain and stress elements and the stress element in the $z$ direction become equal to zero:

$\varepsilon_{x x}=\varepsilon_{y y}$

$\varepsilon_{x y}=\varepsilon_{x z}=\varepsilon_{y z}=0$

$\sigma_{x y}=\sigma_{x z}=\sigma_{y z}=0$

$\sigma_{z z}=0$ whereas the nonzero strain parameters may be expressed as

$\varepsilon_{x x}=\varepsilon_{a}$

$\varepsilon_{z z}=-\frac{2 C_{13}}{C_{33}} \varepsilon_{x x}$

Generally, the polarization vector $\boldsymbol{P}$ is equal to the tensor of piezoelectric coefficients $e_{i j}$ multiplied by the strain tensor $\varepsilon_{k l}$ :

$\left[\begin{array}{c}P_{x} \\ P_{y} \\ P_{z}\end{array}\right]=\left[\begin{array}{cccccc}0 & 0 & 0 & 0 & e_{15} & 0 \\ 0 & 0 & 0 & e_{15} & 0 & 0 \\ e_{31} & e_{31} & e_{33} & 0 & 0 & 0\end{array}\right]\left[\begin{array}{c}\varepsilon_{x x} \\ \varepsilon_{y y} \\ \varepsilon_{z z} \\ 2 \varepsilon_{y z} \\ 2 \varepsilon_{x z} \\ 2 \varepsilon_{x y}\end{array}\right]$

Then, taking advantage of the above relations (7a)-(8b), the only nonzero component of the polarization vector may be expressed in the following form:

$P_{z}=2\left(e_{31}-\frac{C_{13}}{C_{33}}\right) \varepsilon_{x x}$

\section{More natural approach to stresses in nitride structures}

In the above approach proposed by Romanov et al. [7], components of the strain and stress tensors, determined with the aid of the stiffness tensor $C_{i j}$ for the local $0 x y z$ system, are transformed into the global $0 x^{\prime} y^{\prime} z^{\prime}$ system. However, it seems to be more natural to transform first the stiffness tensor $C_{i j}$ from the local coordinate $0 x y z$ system to the global $0^{\prime} x^{\prime} y^{\prime} z^{\prime}$ one and next to use its transformed version $C_{i j}^{\prime}$ to determine directly strain and stress phenomena. Both approaches should give identical results, however, the latter one seems to be more natural and may be successfully used in various special cases.

Generally, the fourth-rank elastic stiffness tensor $C_{t u v w}$ may be transformed into $C_{i j k l}^{\prime}$ one with the aid of the direction cosine matrix $a_{m n}$ :

$C_{i j k l}^{\prime}=a_{i t} a_{j u} a_{k v} a_{l w} C_{t u v w}$

where for the rotation of the Cartesian system around the $0 x$ axis by the angle $\vartheta$ :

$a_{m n}=\left[\begin{array}{ccc}1 & 0 & 0 \\ 0 & \cos \vartheta & -\sin \vartheta \\ 0 & \sin \vartheta & \cos \vartheta\end{array}\right]$ 
The new $C_{i j}^{\prime}$ tensor (in the Voigt notation) contains 20 nonzero and 13 independent elements:

$C_{i j}^{\prime}=\left[\begin{array}{cccccc}C_{11}^{\prime} & C_{12}^{\prime} & C_{13}^{\prime} & C_{14}^{\prime} & 0 & 0 \\ C_{12}^{\prime} & C_{22}^{\prime} & C_{23}^{\prime} & C_{24}^{\prime} & 0 & 0 \\ C_{13}^{\prime} & C_{23}^{\prime} & C_{33}^{\prime} & C_{34}^{\prime} & 0 & 0 \\ C_{14}^{\prime} & C_{24}^{\prime} & C_{34}^{\prime} & C_{44}^{\prime} & 0 & 0 \\ 0 & 0 & 0 & 0 & C_{55}^{\prime} & C_{56}^{\prime} \\ 0 & 0 & 0 & 0 & C_{56}^{\prime} & C_{66}^{\prime}\end{array}\right]$

Its $C_{i j}^{\prime}$ elements are related to the previous $C_{i j}$ ones in a following way:

$C_{11}^{\prime}=C_{11}$

$C_{12}^{\prime}=C_{13} \sin ^{2} \vartheta+C_{12} \cos ^{2} \vartheta$

$C_{13}^{\prime}=C_{13} \cos ^{2} \vartheta+C_{12} \sin ^{2} \vartheta$

$C_{14}^{\prime}=C_{12} \cos \vartheta \sin \vartheta-C_{13} \cos \vartheta \sin \vartheta$

$C_{22}^{\prime}=\left[\left(2 C_{44}+C_{13}\right) / 2\right] \sin ^{2}(2 \vartheta)+C_{33} \sin ^{4} \vartheta$

$$
+C_{11} \cos ^{4} \vartheta
$$

$C_{23}^{\prime}=\left[\left(C_{33}+C_{11}\right) / 4-C_{44}\right] \sin ^{2}(2 \vartheta)+C_{13}\left(\sin ^{4} \vartheta\right.$

$$
\left.+\cos ^{4} \vartheta\right)
$$

$C_{24}^{\prime}=\left\{\left[\left(C_{13}-C_{33}\right) / 2\right] \sin ^{2} \vartheta+\left[\left(C_{11}-C_{13}\right) / 2\right]\right.$

$$
\left.\times \cos ^{2} \vartheta-C_{44} \cos (2 \vartheta)\right\} \sin (2 \vartheta)
$$

$C_{33}^{\prime}=\left[\left(2 C_{44}+C_{13}\right) / 2\right] \sin ^{2}(2 \vartheta)+C_{33} \cos ^{4} \vartheta$

$$
+C_{11} \sin ^{4} \vartheta
$$

$C_{34}^{\prime}=\left\{\left[\left(C_{13}-C_{33}\right) / 2\right] \cos ^{2} \vartheta+\left[\left(C_{11}-C_{13}\right) / 2\right]\right.$

$$
\left.\times \sin ^{2} \vartheta+C_{44} \cos (2 \vartheta)\right\} \sin (2 \vartheta)
$$

$C_{44}^{\prime}=C_{44} \cos ^{2}(2 \vartheta)+\left[\left(C_{33}-2 C_{13}+C_{11}\right) / 4\right] \sin ^{2}(2 \vartheta)$

$C_{55}^{\prime}=C_{44} \cos ^{2} \vartheta+\left[\left(C_{11}-C_{12}\right) / 2\right] \sin ^{2} \vartheta$

$C_{56}^{\prime}=\left[\left(C_{11}-2 C_{44}-C_{12}\right) / 4\right] \sin (2 \vartheta)$

$C_{66}^{\prime}=C_{44} \sin ^{2} \vartheta+\left[\left(C_{11}-C_{12}\right) / 2\right] \cos ^{2} \vartheta$

Then the Hook's law (5) and (4) relations lead to the following set of equations for the stress elements in the global $0 x^{\prime} y^{\prime} z^{\prime}$ system:

$\sigma_{x^{\prime} x^{\prime}}=C_{11}^{\prime} \varepsilon_{x^{\prime} x^{\prime}}+C_{12}^{\prime} \varepsilon_{y^{\prime} y^{\prime}}+C_{13}^{\prime} \varepsilon_{z^{\prime} z^{\prime}}+2 C_{14}^{\prime} \varepsilon_{y^{\prime} z^{\prime}}$

$\sigma_{y^{\prime} y^{\prime}}=C_{12}^{\prime} \varepsilon_{x^{\prime} x^{\prime}}+C_{22}^{\prime} \varepsilon_{y^{\prime} y^{\prime}}+C_{23}^{\prime} \varepsilon_{z^{\prime} z^{\prime}}+2 C_{24}^{\prime} \varepsilon_{y^{\prime} z^{\prime}}$

$\sigma_{z^{\prime} z^{\prime}}=C_{13}^{\prime} \varepsilon_{x^{\prime} x^{\prime}}+C_{23}^{\prime} \varepsilon_{y^{\prime} y^{\prime}}+C_{33}^{\prime} \varepsilon_{z^{\prime} z^{\prime}}+2 C_{34}^{\prime} \varepsilon_{y^{\prime} z^{\prime}}=0(15 \mathrm{c})$

$\sigma_{y^{\prime} z^{\prime}}=C_{14}^{\prime} \varepsilon_{x^{\prime} x^{\prime}}+C_{24}^{\prime} \varepsilon_{y^{\prime} y^{\prime}}+C_{34}^{\prime} \varepsilon_{z^{\prime} z^{\prime}}+2 C_{44}^{\prime} \varepsilon_{y^{\prime} z^{\prime}}=0(15 \mathrm{~d})$

$\sigma_{x^{\prime} z^{\prime}}=2 C_{55}^{\prime} \varepsilon_{x^{\prime} z^{\prime}}+2 C_{56}^{\prime} \varepsilon_{x^{\prime} y^{\prime}}=0$ $\sigma_{x^{\prime} y^{\prime}}=2 C_{56}^{\prime} \varepsilon_{x^{\prime} z^{\prime}}+2 C_{66}^{\prime} \varepsilon_{x^{\prime} y^{\prime}}$

Next, taking into account the condition (3) $\varepsilon_{x^{\prime} y^{\prime}}=0$, we have, from (15e) and (15f):

$\varepsilon_{x^{\prime} z^{\prime}}=0 \quad$ and $\quad \sigma_{x^{\prime} y^{\prime}}=0$

Finally, Eqs. (15c) and (15d) result in the following relations:

$\varepsilon_{z^{\prime} z^{\prime}}^{\prime}=\frac{\left(C_{13}^{\prime} C_{44}^{\prime}-C_{14}^{\prime} C_{34}^{\prime}\right) \varepsilon_{x^{\prime} x^{\prime}}+\left(C_{23}^{\prime} C_{44}^{\prime}-C_{24}^{\prime} C_{34}^{\prime}\right) \varepsilon_{y^{\prime} y^{\prime}}}{C_{34}^{\prime 2}-C_{33}^{\prime} C_{44}^{\prime}}$

$\varepsilon_{y^{\prime} z^{\prime}}^{\prime}=\frac{\left(C_{14}^{\prime} C_{33}^{\prime}-C_{13}^{\prime} C_{34}^{\prime}\right) \varepsilon_{x^{\prime} x^{\prime}}+\left(C_{24}^{\prime} C_{33}^{\prime}-C_{23}^{\prime} C_{34}^{\prime}\right) \varepsilon_{y^{\prime} y^{\prime}}}{C_{34}^{\prime 2}-C_{33}^{\prime} C_{44}^{\prime}}$

\section{Piezoelectric effects in the semi-polar crystal structure}

Following the above calculations, components of the piezoelectric polarization $\boldsymbol{P}_{\mathrm{PZ}}$ may be found from the following relation:

$$
\left[\begin{array}{c}
P_{x^{\prime}} \\
P_{y^{\prime}} \\
P_{z^{\prime}}
\end{array}\right]=\left[\begin{array}{cccccc}
0 & 0 & 0 & 0 & e_{15}^{\prime} & e_{16}^{\prime} \\
e_{21}^{\prime} & e_{22}^{\prime} & e_{23}^{\prime} & e_{24}^{\prime} & 0 & 0 \\
e_{31}^{\prime} & e_{32}^{\prime} & e_{33}^{\prime} & e_{34}^{\prime} & 0 & 0
\end{array}\right]\left[\begin{array}{c}
\varepsilon_{x^{\prime} x^{\prime}} \\
\varepsilon_{y^{\prime} y^{\prime}} \\
\varepsilon_{z^{\prime} z^{\prime}} \\
2 \varepsilon_{y^{\prime} z^{\prime}} \\
2 \varepsilon_{x^{\prime} z^{\prime}} \\
2 \varepsilon_{x^{\prime} y^{\prime}}
\end{array}\right]
$$

where components $e_{i j}^{\prime}$ of the piezoelectric tensor in the $0 x^{\prime} y^{\prime} z^{\prime}$ coordinate system may be written in the following form:

$$
\begin{aligned}
e_{15}^{\prime}= & e_{15} \cos \vartheta \\
e_{16}^{\prime}= & -e_{15} \sin \vartheta \\
e_{21}^{\prime}= & -e_{31} \sin \vartheta \\
e_{22}^{\prime}= & -\left[e_{33} \sin ^{2} \vartheta+\left(e_{31}+2 e_{15}\right) \cos ^{2} \vartheta\right] \sin \vartheta \\
e_{23}^{\prime}= & -\left[e_{31} \sin ^{2} \vartheta+\left(e_{33}-2 e_{15}\right) \cos ^{2} \vartheta\right] \sin \vartheta \\
e_{24}^{\prime}= & -\left[-e_{15} \cos (2 \vartheta)+\left(e_{31}-e_{33}\right) \sin ^{2} \vartheta\right] \cos \vartheta \\
e_{31}^{\prime}= & e_{31} \cos \vartheta \\
e_{32}^{\prime}= & {\left[e_{31} \cos ^{2} \vartheta+\left(e_{33}-2 e_{15}\right) \sin ^{2} \vartheta\right] \cos \vartheta } \\
e_{33}^{\prime}= & {\left[e_{33} \cos ^{2} \vartheta+\left(e_{31}+2 e_{15}\right) \sin ^{2} \vartheta\right] \cos \vartheta } \\
e_{34}^{\prime}= & \left\{\left(e_{31}-e_{33}\right) \cos { }^{5} \vartheta-e_{15}[\cos (2 \vartheta)\right. \\
& \left.\left.+0.5 \sin ^{2}(2 \vartheta)\right] \cos \vartheta\right\} \sin \vartheta
\end{aligned}
$$


Table 1 Values of parameters used in the calculations

\begin{tabular}{|c|c|c|c|c|c|c|}
\hline Parameter & $\mathrm{GaN}$ & AlN & $\mathrm{InN}$ & Ref. & $\mathrm{In}_{0.2} \mathrm{Ga}_{0.8} \mathrm{~N}$ & $\mathrm{In}_{0.3} \mathrm{Ga}_{0.7} \mathrm{~N}$ \\
\hline$a(\mathrm{~nm})$ & 3.189 & 3.112 & 3.545 & {$[13]$} & 3.2602 & 3.2958 \\
\hline$c(\mathrm{~nm})$ & 5.185 & 4.982 & 5.703 & {$[13]$} & 5.2886 & 5.3404 \\
\hline$E_{G}(\mathrm{eV})$ & 3.4 & 6.0 & 0.8 & {$[4]$} & 2.65 & 2.32 \\
\hline$C_{11}(\mathrm{GPa})$ & 390 & 396 & 223 & {$[13]$} & 356.6 & 339.9 \\
\hline$C_{12}(\mathrm{GPa})$ & 145 & 137 & 115 & {$[13]$} & 139 & 136 \\
\hline$C_{13}(\mathrm{GPa})$ & 106 & 108 & 92 & {$[13]$} & 103.2 & 101.8 \\
\hline$C_{33}(\mathrm{GPa})$ & 398 & 373 & 224 & {$[13]$} & 363.2 & 345.8 \\
\hline$C_{44}(\mathrm{GPa})$ & 105 & 116 & 48 & {$[13]$} & 93.6 & 87.9 \\
\hline$e_{33}\left(\mathrm{~cm}^{-2}\right)$ & 0.667 & 1.505 & 0.815 & {$[8]$} & 0.697 & 0.711 \\
\hline$e_{31}\left(\mathrm{~cm}^{-2}\right)$ & -0.338 & -0.533 & -0.412 & {$[8]$} & -0.353 & -0.360 \\
\hline$e_{15}\left(\mathrm{~cm}^{-2}\right)$ & -0.167 & -0.351 & -0112 & {$[8]$} & -0.156 & -0.151 \\
\hline$P_{\mathrm{SP}}\left(\mathrm{cm}^{-2}\right)$ & -0.034 & -0.042 & -0.090 & {$[8]$} & -0.0417 & -0.0443 \\
\hline$\varepsilon$ & 10.4 & 8.5 & 15.3 & {$[4]$} & 11.38 & 11.87 \\
\hline$m_{\mathrm{e}}^{*}\left(m_{0}\right)$ & 0.21 & 0.32 & 0.07 & [13] & 0.182 & 0.168 \\
\hline$m_{\mathrm{hh}}^{*}\left(m_{0}\right)$ & 1.89 & 3.57 & 1.56 & {$[13]$} & 1.822 & 1.790 \\
\hline
\end{tabular}

Then Eq. (18) leads to the following vector of the piezoelectric polarization $\boldsymbol{P}_{\mathrm{PZ}}$ :

$\left[\begin{array}{c}P_{x^{\prime}} \\ P_{y^{\prime}} \\ P_{z^{\prime}}\end{array}\right]=\left[\begin{array}{c}2 e_{15}^{\prime} \varepsilon_{x^{\prime} z^{\prime}}+2 e_{16}^{\prime} \varepsilon_{x^{\prime} y^{\prime}} \\ e_{22}^{\prime} \varepsilon_{x^{\prime} x^{\prime}}+e_{22}^{\prime} \varepsilon_{y^{\prime} y^{\prime}}+2 e_{22}^{\prime} \varepsilon_{y^{\prime} z^{\prime}} \\ e_{31}^{\prime} \varepsilon_{x^{\prime} x^{\prime}}+e_{32}^{\prime} \varepsilon_{y^{\prime} y^{\prime}}+2 e_{34}^{\prime} \varepsilon_{y^{\prime} z^{\prime}}\end{array}\right]$

As one can see, because from (3) $\varepsilon_{x^{\prime} y^{\prime}}=0$ and from (13) $\varepsilon_{x^{\prime} z^{\prime}}=0$, the $x^{\prime}$-component of the piezoelectric vector disappears.

For the nitride $\operatorname{In}_{x} \mathrm{Ga}_{1-x} \mathrm{~N}$ compound, the spontaneous polarization is expressed as [8]:

$$
P_{\mathrm{SP}}\left[C / m^{2}\right]=-0.042 x-0.034(1-x)+0.0378 x(1-x)
$$

In the case of the semipolar crystal orientation, the polarization component perpendicular to the layer depends on the inclination angle $\vartheta$ :

$P_{\mathrm{SP}, z}=P_{\mathrm{SP}} \cos \vartheta$

The total polarization is a sum of the spontaneous and piezoelectric polarizations:

$\boldsymbol{P}=\boldsymbol{P}_{\mathrm{SP}}+\boldsymbol{P}_{\mathrm{PZ}}$

Polarization within a bulk material does not practically have any impact on its properties. But in a layered structure, it is followed by some polarization discontinuities at layer interfaces which lead to accumulation of electrical charges. In a multilayered structure, the electric field within the $j$ th layer is reduced to zero when total polarizations $P_{i}$ of these layers fulfills the following condition:

$\sum_{i} \frac{l_{i}}{\kappa_{i}}\left(P_{i}-P_{j}\right)=0$

where $l_{i}$ and $\kappa_{i}$ are thicknesses and permittivities of successive layers. It is easy to conclude from the above equation that electric fields will be equal to zero in all layers when polarizations within them are equal to one another. Hence polarization-induced effects may be eliminated not only for polarizations reduced to zero in all the layers, but also for the same polarizations in successive structure layers.

The above equations have been derived for the semipo$\operatorname{lar}(1 \underline{10 l)}$ orientations for $l=1,2,3, \ldots$ Following [7], we assume applicability of this approach also for other orientations, the $(1 \underline{1} 2 l)$ orientation in particular.

\section{Designing of nitride LEDs with reduced polarization effects}

The model presented in the previous sections has been used to simulate performance characteristics of $I n G a N / G a N$ LEDs emitting green radiation. A typical nitride LED structure is composed of the following layer sequence: $n$-type substrate, QW barrier, QW, QW barrier, electron-blocking layer, and $p$-type layer, all manufactured on a polar, nonpolar or semipolar substrates. In the calculations, the 3-nm InGaN QW surrounded by $12-\mathrm{nm}$ GaN barriers is assumed as the LED active region. Used values of model parameters are listed in Table 1. 
The main goal of this paper is to propose a method, which may be used to overcome polarization problems in semipolar nitride LEDs emitting green radiation. The method is expected to lead to such a structure of InGaN/GaN QW LEDs, which enables efficient emission of green $(\lambda \approx 520 \mathrm{~nm})$ radiation and exhibits reduced unwanted QCSE polarization phenomena. To this end, three conditions should be fulfilled. First, efficient emission requires structures with a low density of defects, which may be achieved using growth planes characterized by low values of the Miller-Bravais indices [7]. Next, emission of green radiation may be achieved in InGaN/GaN QWs characterized by a relative high amount of the Indium content. And finally, reduced polarization effects require similar values of polarizations in both $\mathrm{QW}$ and barrier materials. Let us now consider realization of the above conditions.

Zhao et al. [14] have presented results of a comparative analysis of various substrate orientations used to produce nitride emitters of long-wavelength radiation, i.e., lightemitting diodes (LEDs) and diode lasers (DLs). According to their results, the highest Indium incorporation necessary to obtain green radiation of longer wavelengths has been recorded for the (1122) GaN orientation, although the (2021) and partly the (2021) orientations also enable reaching InGaN layers of a high Indium mole fractions. Analogous results, i.e., the highest InN mole fraction, have been also determined for the inclination angle $58^{\circ}$ (i.e., for the (1122) orientation) using a simplified theoretical model [15]. The $p-n$ junction built-in electric field has been found to oppose the polarization-related electric field in the quantum wells (QWs) on the (2021)-oriented substrate whereas both the above fields have the same directions within the QWs on the (1122) and the (2021) substrates. Because of that, potential profiles within the QWs on the (2021) substrate exhibit relatively flat shape whereas profitable triangular potential profiles are found in QWs on the (1122) and the (2021) substrates. As a result, LEDs created on the (1122) plane emit radiation of distinctly longer wavelengths [14] than those produced on the (2021) substrate. In our model, the current spreading is connected only with polarization changes. Hsu et al. [16] have reported formation of misfit dislocations (MDs) in (1122) InGaN layers grown on $\mathrm{GaN}$ substrates due to stress relaxation, however, they have concluded that similar mechanisms for MDs formation have been expected for other semi-polar orientations. Besides, (1122) oriented films appear to be mostly smooth [9]. Effective long-wavelength emission of InGaN/GaN LEDs grown on the (2021) substrate and on the (2021) substrate has been reported in many papers (see, e.g., [17-19] and [20-22], respectively). However, taking into account aggregate results of the above analysis, we have decided to choose the (1122) InGaN/GaN LEDs. Efficient long-wavelength emission of these devices has been also reported many times (see,

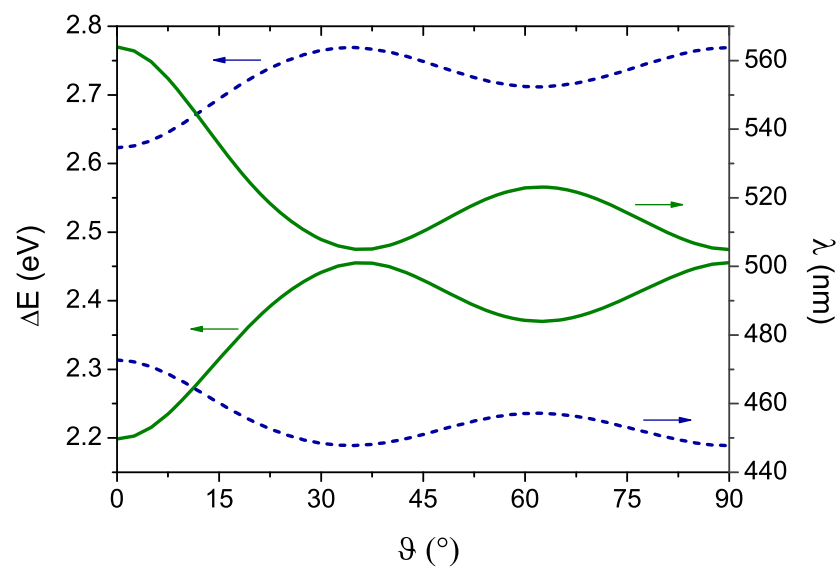

Fig. 4 Energy distance $\Delta E$ between the electron and the heavyhole fundamental states and corresponding wavelength $\lambda$ of emitted radiation plotted as a function of the inclination angle $\vartheta$ for the $\mathrm{In}_{0.2} \mathrm{Ga}_{0.8} \mathrm{~N} / \mathrm{GaN} \mathrm{QW}$ (dashed lines) and the $\mathrm{In}_{0.3} \mathrm{Ga}_{0.7} \mathrm{~N} / \mathrm{GaN} \mathrm{QW}$ (solid lines)

e.g., [23-27]) and their structures seem to have currently more perspective advantages as green emitters [28] among reported orientations to date. In particular, expected highpower green $(516 \mathrm{~nm})$ emission has been reported by Sato et al. [29] from the InGaN/GaN multi-quantum-well LED manufactured on the (1122) GaN substrate.

Figure 4 shows a distance $\Delta E$ between energy levels of electron and heavy-hole fundamental QW states as a function of the inclination angle $\vartheta$ determined for both the $\mathrm{In}_{0.2} \mathrm{Ga}_{0.8} \mathrm{~N} / \mathrm{GaN}$ QW and the $\mathrm{In}_{0.3} \mathrm{Ga}_{0.7} \mathrm{~N} / \mathrm{GaN}$ QW. Electron recombinations with both light holes and split-off holes are neglected because of their shorter emission wavelengths [30]. Besides, also a wavelength $\lambda$ of emitted radiation is shown in Fig. 4. As one can see, desired emission of green radiation is possible in LEDs with the $\mathrm{In}_{0.3} \mathrm{Ga}_{0.7} \mathrm{~N} / \mathrm{GaN}$ QWs. Since the heavy-hole band has the lowest energy in the valence band, radiative transitions between electrons and heavy holes gives the longest possible wavelength. However, it should be remembered that efficiency of semipolar InGaN/GaN QW LEDs drastically decreases at longer wavelengths due to difficulties associated with a growth of InGaN layers of high Indium composition [1]. This growth required relatively low growth temperatures, which often results in a degraded crystal quality [2].

Electric field generated in a quantum well is proportional to a difference $\Delta P$ in total polarizations of the quantum well material and the barrier. For the InGaN/GaN QWs, a complete elimination of the QCSE, achieved for $\Delta P=0$, is possible not only for the nonpolar crystal orientation $\left(\vartheta=90^{\circ}\right)$, which is obvious, but also for one its orientation defined in this case by the inclination angle about $34^{\circ}$. An increase in Indium content in QW results in shifting this angle toward somewhat larger $\vartheta$. For $\vartheta$ equal to about $62^{\circ}$, a change $\Delta P$ of the total polarization at the interface between the InGaN 



Fig. 5 Fundamental-state electron (a) and heavy-hole (b) wave functions within the $\operatorname{In}_{0.2} \mathrm{Ga}_{0.8} \mathrm{~N} / \mathrm{GaN} \mathrm{QW}$ plotted for various inclination angles $\vartheta$

quantum well and the GaN barrier exhibits some maximum, which however is about twice lower than that in the case of polar $(\vartheta=0)$ orientation. It confirms anticipated suppression of polarization effects in all possible semipolar LEDs as compared with polar ones. Besides, a change of $\Delta P$ from positive to negative values for lower $\vartheta$ is followed by inverted slope of QW band edges. The (1122) crystal orientation does not enable fulfilling the condition $\Delta P=0$, but fortunately gives reduction of around $50 \%$ in comparison with the $c$ orientation.

A change of the electron $\left(\psi_{\mathrm{e}}\right)$ and the heavy-hole $\left(\psi_{\mathrm{hh}}\right)$ wave functions within the $3-\mathrm{nm} \mathrm{In}_{0.2} \mathrm{Ga}_{0.8} \mathrm{~N} / \mathrm{GaN}$ QW with an increase in the inclination angle $\vartheta$ is shown in Figs. 5a and $5 \mathrm{~b}$, respectively. As expected, perfect overlapping of electron and heavy-hole wave functions, desired for enhanced carrier recombination, is seen for the non-polar $(\vartheta=$ $90^{\circ}$ ) crystal orientation. For lower inclination angles $\vartheta$, i.e. for semi-polar orientations, the overlapping depends on a distance of $\vartheta$ from its value (about $34^{\circ}$ ) corresponding to

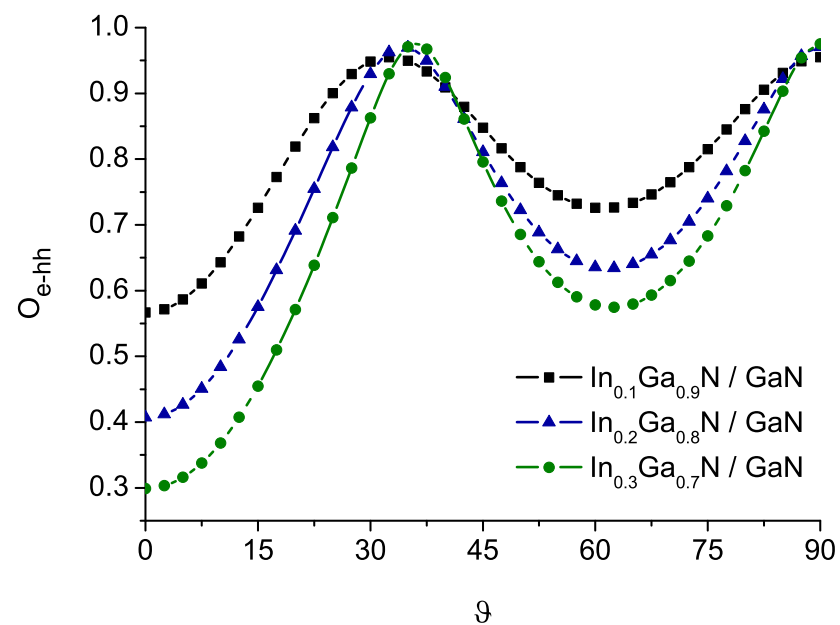

Fig. 6 Overlapping $O_{\mathrm{e}-\mathrm{hh}}$ of the conduction-band electron $\Psi_{\mathrm{e}}$ and the valence-band heavy-hole $\Psi_{\text {hh }}$ wave functions within three various $3-\mathrm{nm}$ InGaN/GaN quantum wells as a function of the inclination angle $\vartheta$ of their substrate orientation

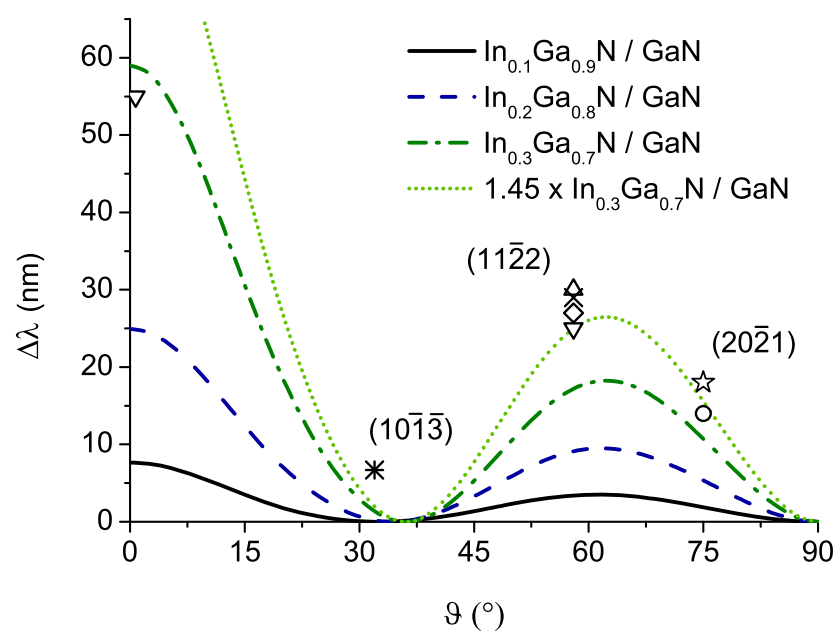

Fig. 7 Theoretically determined maximal shift $\Delta \lambda$ of an emission spectrum associated with the QCSE within the 3-nm QWs as a function of the inclination angle $\vartheta$ for the $\operatorname{In}_{0.1} \mathrm{Ga}_{0.9} \mathrm{~N} / \mathrm{GaN} \mathrm{QW}$ (solid line), the $\mathrm{In}_{0.2} \mathrm{Ga}_{0.8} \mathrm{~N} / \mathrm{GaN} \mathrm{QW}$ (dashed line), and the $\mathrm{In}_{0.3} \mathrm{Ga}_{0.7} \mathrm{~N} / \mathrm{GaN} \mathrm{QW}$ (dash-dot line). The dotted line presents results determined for the $\mathrm{In}_{0.3} \mathrm{Ga}_{0.7} \mathrm{~N} / \mathrm{GaN} \mathrm{QW}$ multiplied by 1.45 to match experimental values. Points correspond to experimental values obtained for LEDs emitting green light $[20,21,23,26,27,29,31]$

the same polarizations in both the quantum well and the barrier (Fig. 6).

Intensity of the band-to-band recombination of electrons and heavy holes within 3-nm InGaN/GaN QWs is proportional to the overlapping $O_{\mathrm{e}-\mathrm{hh}}$ (Fig. 6) of the conductionband electron $\Psi_{\mathrm{e}}$ and the valence-band heavy-hole $\Psi_{\mathrm{hh}}$ wave functions. As one can see, the above overlapping for the considered $\mathrm{In}_{0.3} \mathrm{Ga}_{0.7} \mathrm{~N} / \mathrm{GaN}$ QW is increased from about 0.30 for the polar $(\vartheta=0)$ crystal orientation to about 0.58 for the semipolar $(11 \underline{22})$ orientation $\left(\vartheta=58^{\circ}\right)$. 
Taking into account all the above facts, the $\operatorname{In}_{0.3} \mathrm{Ga}_{0.7} \mathrm{~N} /$ GaN QW LEDs grown on the semi-polar (112) plane seems to be the most promising LED structure emitting effectively desired green radiation.

In Fig. 7, a dependence on an inclination angle of maximal shifts $\Delta \lambda$ of an emission spectrum as a result of the QCSE determined by the model for three $\mathrm{In}_{x} \mathrm{Ga}_{1-x} \mathrm{~N} / \mathrm{GaN} \mathrm{QWs}$ is compared with some experimental values. The above experimental shifts found for semipolar $\mathrm{In}_{x} \mathrm{Ga}_{1-x} \mathrm{~N} / \mathrm{GaN}$ QWs (varying slightly according to $x$ values, QW widths as well as operation currents) are systematically larger than values determined theoretically. This can be explained by inhomogeneity of In content [32] within InGaN/GaN QWs which gives additional blue-shift, especially for low driving currents, due to the band-filling effect. Therefore, theoretical shifts have been used to match experimental results. As one can see in Fig. 7, those theoretical values are in a quantitative agreement with experiments, which serves as a validation of our model.

\section{Conclusions}

In nitride light-emitting diodes (LEDs) manufactured on standard polar substrates, unavoidable polarization effects hamper in reaching their efficient long-wavelength emission. Those effects may be completely avoided in non-polar crystallographic orientations, but then there are problems with reaching relatively high Indium contents in InGaN active regions necessary for the green emission. Therefore, the semipolar crystallographic substrate orientations should be used. Following comparative analysis given in the above fifth section, we may conclude that, from considered crystal orientations, the (1122) crystallographic substrate orientation (inclination angle of $58^{\circ}$ ) seems to be the most promising because:

- it makes possible an increased Indium incorporation efficiency enabling efficient green emission,

- LEDs created on the (1122) plane emit radiation of longer green wavelengths than those produced on other substrates,

- it is possible to suppress in these LEDs polarization effects, and

- (1122) oriented films appear to be mostly smooth [9].

Therefore, the $\operatorname{In}_{0.3} \mathrm{Ga}_{0.7} \mathrm{~N} / \mathrm{GaN}$ QW LED grown on the semipolar (1122) substrate (inclination angle of $58^{\circ}$ ) has been found as currently optimal LED structure emitting a green radiation, although similar LEDs manufactured on the (2021) and the (2021) substrates also exhibit comparable performance.

Acknowledgements We acknowledge the financial support of the European Union within European Regional Development Fund, through Grant Innovative Economy (POIG.01.03.01-00-159/08, "InTechFun").
Open Access This article is distributed under the terms of the Creative Commons Attribution License which permits any use, distribution, and reproduction in any medium, provided the original author(s) and the source are credited.

\section{References}

1. S. Nakamura, Mater. Res. Soc. Bull. 34, 101 (2009)

2. D.F. Feezell, M.C. Schmidt, S.P. DenBaars, S. Nakamura, Mater. Res. Soc. Bull. 34, 318 (2009)

3. T.K. Sharma, E. Towe, J. Appl. Phys. 107, 024516 (2010)

4. H. Morkoç, Materials Properties. Handbook of Nitride Semiconductors and Devices, vol. 1 (Wiley-VCH, Weinheim, 2008)

5. M.T. Hardy, D.F. Feezell, S.P. DenBaars, S. Nakamura, Mater. Today 14, 408 (2011)

6. T.J. Baker, B.A. Haskell, F. Wu, P.T. Fini, J.S. Speck, S. Nakamura, Jpn. J. Appl. Phys. 44, L920 (2005)

7. A.E. Romanov, T.J. Baker, S. Nakamura, J.S. Speck, J. Appl. Phys. 100, 023522 (2006)

8. F. Bernardini, in Nitride Semiconductor Devices: Principles and Simulation, ed. by J. Piprek (Wiley-VCH, Weinheim, 2007)

9. H. Masui, S.C. Cruz, S. Nakamura, S.P. DenBaars, J. Electron. Mater. 68, 756 (2009)

10. Q.Y. Wei, T. Li, Z.H. Wu, F.A. Ponce, Phys. Status Solidi A 207, 2226 (2010)

11. R. Dwiliński, R. Doradziński, J. Garczyński, L. Sierzputowski, R. Kucharski, M. Rudziński, M. Zając, R. Kudrawiec, Phys. Status Solidi C 6, 2661 (2009)

12. J.F. Nye, Physical Properties of Crystals (Oxford University Press, New York, 1985)

13. I. Vurgaftman, J.R. Meyer, Electron bandstructure parameters, in Nitride Semiconductor Devices: Principles and Simulation, ed. by J. Piprek (Wiley-VCH, Weinheim, 2007)

14. Y. Zhao, Q. Yan, C.-Y. Huang, S.-C. Huang, P.S. Hsu, S. Tanaka, C.-C. Pan, Y. Kawaguchi, K. Fujito, C.G. Van de Walle, J.S. Speck, S.P. DenBaars, S. Nakamura, D. Feezell, Appl. Phys. Lett. 100, 201108 (2012)

15. M.V. Durnev, A.V. Omelchenko, E.V. Yakovlev, I.Yu. Evstratov, S.Yu. Karpov, Appl. Phys. Lett. 97, 051904 (2010)

16. P.S. Hsu, E.C. Young, A.E. Romanov, K. Fujito, S.P. DenBaars, S. Nakamura, J.S. Speck, Appl. Phys. Lett. 99, 081912 (2011)

17. Y. Zhao, S. Tanaka, Q. Yan, C.-Y. Huang, R.B. Chung, C.-C. Pan, K. Fujito, D. Feezell, C.G. Van de Walle, J.S. Speck, S.P. DenBaars, S. Nakamura, Appl. Phys. Lett. 99, 051109 (2011)

18. Y. Zhao, S. Tanaka, C.-C. Pan, K. Fujito, D. Feezell, J.S. Speck, S.P. DenBaars, S. Nakamura, Appl. Phys. Express 4, 082104 (2011)

19. C.-Y. Huang, M.T. Hardy, K. Fujito, D.F. Feezell, J.S. Speck, S.P. DenBaars, S. Nakamura, Appl. Phys. Lett. 99, 241115 (2011)

20. Y. Enya, Y. Yoshizumi, T. Kyono, K. Akita, M. Ueno, M. Adachi, T. Sumitomo, S. Tokuyama, T. Ikegami, K. Katayama, T. Nakamura, Appl. Phys. Express 2, 082101 (2009)

21. S. Yamamoto, Y. Zhao, C.-C. Pan, R.B. Chung, K. Fujito, J. Sonoda, S.P. DenBaars, S. Nakamura, Appl. Phys. Express 3, 122102 (2010)

22. T. Kyono, Y. Yoshizumi, Y. Enya, M. Adachi, S. Tokuyama, M. Ueno, K. Katayama, T. Nakamura, Appl. Phys. Express 3, 011003 (2010)

23. M. Funato, M. Ueda, Y. Kawakami, Y. Narukawa, T. Kosugi, M. Takahashi, T. Mukai, Jpn. J. Appl. Phys. 45, L659 (2006)

24. H. Zhong, A. Tyagi, N.N. Fellows, R.B. Chung, M. Saito, K. Fujito, J.S. Speck, S.P. DenBaars, S. Nakamura, Electron. Lett. 43, 825 (2007) 
25. H. Sato, R.B. Chung, H. Hirasawa, N. Fellows, H. Masui, F. Wu, M. Saito, K. Fujito, J.S. Speck, S.P. DenBaars, S. Nakamura, Appl. Phys. Lett. 92, 221110 (2008)

26. A. Strittmatter, J.E. Northrup, N.M. Johnson, M.V. Kisin, P. Spiberg, H. El-Ghoroury, A. Usikov, A. Syrkin, Phys. Status Solidi, B Basic Res. 248, 561 (2011)

27. A. Strittmatter, M. Teepe, Z. Yang, C. Chua, J. Northrup, N.M. Johnson, P. Spiberg, R.G.W. Brown, V. Ivantsov, A. Syrkin, L. Shapovalov, A. Usikov, Phys. Status Solidi C 7, 1814 (2010)

28. H. Masui, S. Nakamura, S.P. DenBaars, U.K. Mishra, IEEE Trans. Electron Devices 57, 88 (2010)
29. H. Sato, A. Tyagi, H. Zhong, N. Fellows, R.B. Chung, M. Saito, K. Fujito, J.S. Speck, S.P. DenBaars, S. Nakamura, Phys. Status Solidi (RRL)—Rapid Res. Lett. 1, 162 (2007)

30. I. Vurgaftman, J.R. Meyer, J. Appl. Phys. 94, 3675 (2003)

31. R. Sharma, P. Pattsison, H. Masui, R. Farrell, T. Baker, B. Haskell, F. Wu, S. DenBaars, J. Speck, S. Nakamura, Appl. Phys. Lett. 87, $231110(2005)$

32. T. Li, E. Hahn, D. Gerthsen, A. Rosenauer, A. Strittmatter, L. ReiBmann, D. Bimberg, Appl. Phys. Lett. 86, 241911 (2005) 